\title{
Occupational Exposure to Paints Causes Impairment of Kidney Functions
}

\author{
Saeed S. Al-Ghamdi \\ Department of Pharmacology and Toxicology, Faculty of Medicine, Umm Al-Qura University, Makkah, Kingdom of Saudi Arabia. \\ Email: dr.s.s.alghamdi@gmail.com
}

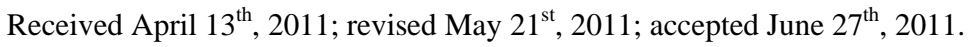

\begin{abstract}
It has been suggested that exposure to organic solvents may have a role in the impairment of kidney function that may progress to kidney failure. However, this has never been evaluated with an appropriate analytical study of the kidney functions of those people who are chronically exposed to these chemicals. This study was designed to measure the kidney function of car painters in the city of Makkah, Saudi Arabia. Fifty workers were selected at random for this study and compared to thirty male medical students who were taken as a control group. Blood samples were collected for the analysis of kidney function. The levels of blood urea nitrogen (BUN), creatinine, and uric acid were scientifically higher in the tested group compared to the control group. In addition to this, the levels of these parameters were significantly higher in the serum of car painters who worked in this industry for more than ten years compared to painters who worked for less than ten years. Moreover, the number of car painters who were not using protective gloves and masks during working hours were 43 and the number of car painters who visited specialized clinics because of kidney problems were 45 of the 50 tested volunteers. These findings support the hypothesized association of solvent exposure with the development of chronic renal failure. They should prompt clinicians to give greater attention to patients' occupational exposures. Routine monitoring of kidney functions and the use of protective materials are of greater importance to minimize the occupational diseases caused by organic solvents.
\end{abstract}

Keywords: Organic Solvents, Uric Acid, Creatinine, BUN, Kidney

\section{Introduction and Methods}

An occupational disease is any chronic ailment that occurs as a result of work or occupational activity. It is an aspect of occupational safety and health, typically identified when it is shown that it is more prevalent in a given body of workers than in the general population, or in other worker populations.

Occupational renal diseases provide models for understanding environmental renal diseases. Kidney damage in few workers induced by heavy exposure to identifiable toxins indicates what to expect among the large population exposed to low levels of organic solvents dispersed in the larger environment [1,2]. Organic solvents are a class of chemical substances that are widely used in large volume in various manufacturing products including the paints. The possible role of organic solvent exposure in the development and/or the progression of chronic renal failure still is a controversial scientific issue more than forty years after the earliest study by Beirne and Brennan [3].
Many epidemiological and recent experimental studies were conducted to prove the effects of these chemicals on kidneys and define the mechanism of action [4-8]. Although some of these studies were criticized for their methodological weaknesses with respect to sample size and inaccuracy in case of definition or exposure assessment, most of them showed significant associations of solvent exposure with various types of kidney disorders [9-13]. These studies, however, never tested the kidney function of people with occupational chronic exposure to organic solvents. I therefore investigated the effect of organic solvent exposures in a cohort of fifty volunteer car painters with the mean of the serum level of uric acid, creatinine and blood urea nitrogen (BUN).

\section{Materials and Methods}

\subsection{Exposure Assessment}

Fifty male volunteer painters were asked about their lifetime occupational activities by trained interviewers using a questionnaire designed for this purpose. For each vol- 
unteer; age, nationality, duration of exposure, health status, and use of protective materials were recorded. Working conditions and frequency of use (every day, at least once a week, occasionally) were also recorded. Thirty male volunteer medical students were used as a negative control for this study.

\subsection{Kidney Function Analysis}

Blood samples were collected for measuring the levels of uric acid, BUN, and creatinine using the Dimension Xpand plus instrument, Dade behring, USA.

\subsection{Statistical Analysis}

Results are presented as mean \pm S.E.M. Means were obtained from six rats from each group. Statistical analysis was performed using one way ANOVA (Kruskal Wallis test) except for the diuretic activity where the Mann Whitney test was used, and the statistical significance was assured when $P<0.05$.

\section{Results}

\subsection{Blood Analysis}

Kidney function tested for the uric acid, creatinene and BUN all showed significantly higher $(P<0.05)$ levels of these parameters in the serum of the car painters compared to the control group (Figure 1).

\subsection{Duration of Exposure}

Analysis of the car painters' duration of exposure shows that the level of the uric acid, creatinine, and BUN in the serum of the tested volunteers who worked for more than ten years in the car painting industry were significantly higher $(P<0.05)$ than those who spent less than ten years in the industry (Figure 2).

\subsection{Use of Protective Materials}

According to the questionnaire records, only seven out of fifty volunteer car painters were using protective gloves and masks during their working hours (Figure 3).

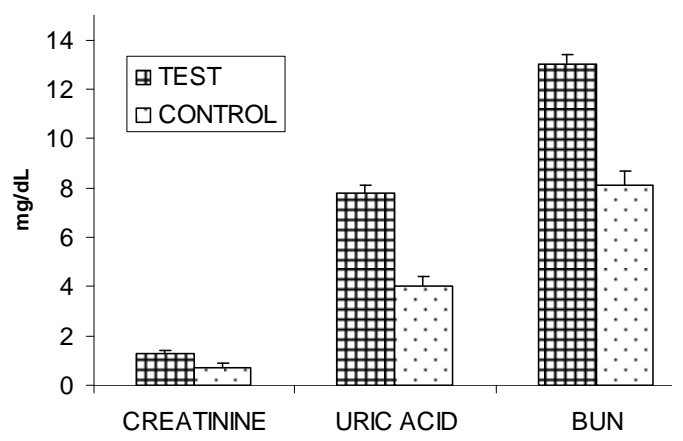

Figure 1. Serum levels of the uric acid, creatinine, and BUN of the studied groups.

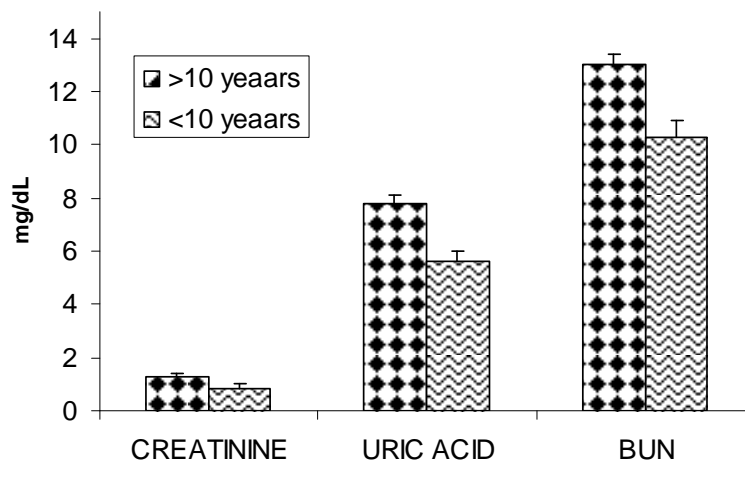

Figure 2. Comparing the levels of serum uric acid, createnine and BUN in car painters who were exposed for more than ten years with those who were exposed for less than ten years.

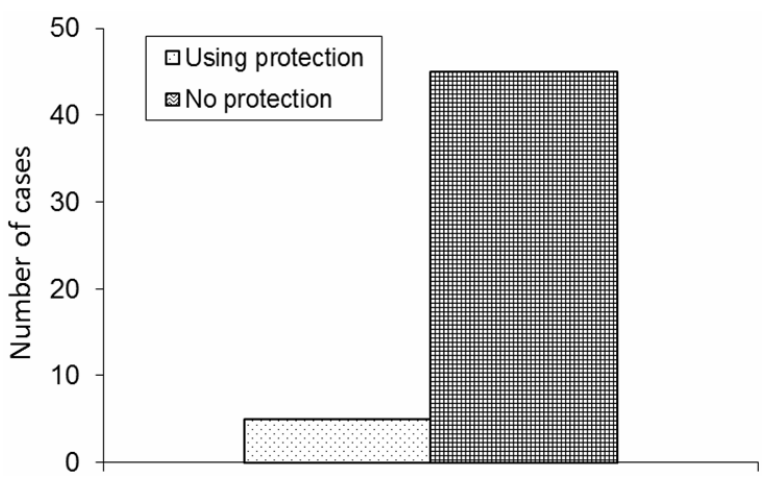

Figure 3. Numbers of painters who were using gloves and masks during work hours.

\subsection{Complaining of Kidney Disorders}

According to the questionnaire records, car painters who visited hospital complaining of kidney pain were higher: 43 out of 50 (Figure 4).

\section{Discussion}

Due to their high lipophilicity, organic solvents may cause physiochemical damage to the renal glomeruli and tubule [14]. Moreover, short-term exposure to these solvents have been shown to cause tubular necrosis, a life-threatening situation characterized by oliguria and azotemia $[15,16]$. By observational assessment of the work place, I noticed that all areas I visited were semi-closed with no adequate ventilation, which meant that painters' exposure to solvents exceeded the threshold limit value recommended for the mixture by the American Conference of Governmental Industrial Hygienists (ACGIH).

Several recent studies have suggested that a relationmay exist between exposure to occupational organic solvents (gasoline, gasoline-based paint spray, jet fuel, mineral turpentine) and diseases of the kidney, particularly malignancy and various forms of glomerulonephritis [17- 


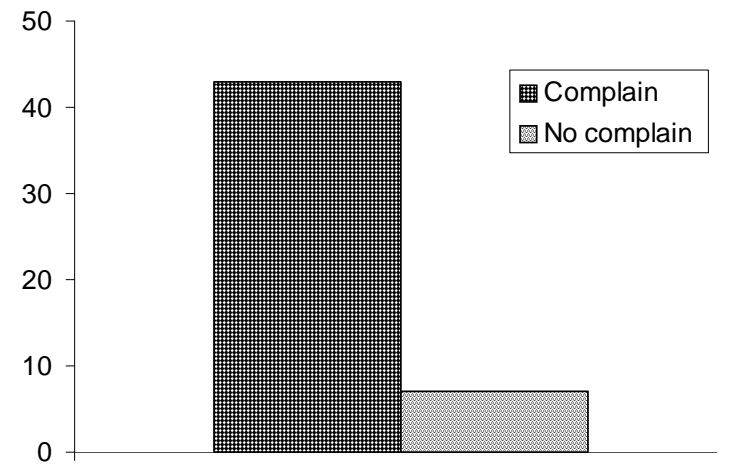

Figure 4. Number of car painters in the tested group who complained of kidney pain.

21]. Retrospective exposure assessment was a critical issue in many of the previous studies. My method is based on a detailed and structured questionnaire and blind evaluation of exposures by experienced car painters and has proven to be very reliable. An important strength of this study, is that I assessed the kidney function of people who were chronically exposed to paints that contain organic solvents and noticed the higher levels of serum creatinine, uric acid and BUN of the painter over the control. My findings are strongly supported by recording the painters who visited urologists for kidney problems. These findings are independent of age and ethnicity.

Different mechanisms of action are probably responseble for the initial events leading to renal tubular cell damage. Unfortunately, for the majority of solvents, the early critical effects are still speculative. The high serum levels of creatinine, uric acid and BUN are considered to be indicators for the damaging of the tubular epithelium. Fortunately, nephrotoxicity caused by organic solvents is reversible once the exposure has ceased. The damaging of tubular epithelium can be recovered, where it takes about three weeks for the tubular epithelium to regenerate. Recovery may be complete, but depending on the severity of the intoxication and the previous status of renal function, some degree of renal insufficiency may persist [13].

The solvent exposure was associated with increasedbaseline proteinuria and the relationship with end-stage renal disease (ESRD) [18]. This relationship tends to weaken after adjustment and suggests that solvent nephrotoxicity may be mediated partly by enhancing proteinuria, a major risk factor for kidney disease progression. In support of this hypothesis, several occupational studies have shown that workers who were exposed to various types of solvents (both aliphatic and aromatic hydrocarbons) had higher levels of albuminuria as well as low molecular weight proteinuria, which is considered to be a marker of tubular injury, as compared with non-exposed workers [19-21].

The limitation of this study, which should be noted, is urine analyses for protein because of technical reasons. However, previous cohort and cross-sectional studies [22-24], which have proven the association between chronic exposure to solvents and albuminurea seem to be adequate. Occupational renal diseases are important because they are entirely preventable. For this reason, the majority of car painters in this study who were not using the protective materials during painting complained of kidney problems and visited specialist clinics for consultation. In addition, renal disease produced by heavy exposure to industrial toxins by relatively few workers provides a clinical basis for understanding the consequences of low-dose exposure to the general population from environmental pollutants. The known toxins that produce kidney disease in the workplace are limited to the heavy metals plus silica and certain organic compounds, particularly halogenated hydrocarbons, employed as Industrial solvents [16]. However, the absence of information on the adverse renal effects of other industrial pollutants does not prove that no such effects occur. In this study, the relationship between the background of the painters and the impairment of the kidney function were not significant. Even though, the probability that the genetic basis of individual susceptibility can not be neglected. The contribution of organic solvents to renal disease of painters based on individual susceptibility may become clearer after certain genetic polymorphism investigations.

\section{Conclusions}

It can be concluded that these results corroborate with an association between renal disorders and exposure to organic solvents. They provide new evidences of the potential role of solvents in the development of kidney diseases. This might be prevented by using protective materials and carrying out routine tests of kidney function.

\section{Acknowledgments}

This study was supported by a grant from the Center for Medical and Medical Sciences Researches, Umm Al-Qura University.

\section{REFERENCES}

[1] T. Ehrenreich, "Renal Disease from Exposure to Solvents,” Annals of Clinical and Laboratory Science, Vol. 7, No. 1, 1977, pp. 6-16.

[2] Wedeen, "Occupational and Environmental Renal Disease,” Seminars in Nephrology, Vol. 17, No. 1, 1997, pp. 46-53.

[3] G. J. Beirne and J. T. Brennan, "Glomerulonephritis Associated with Hydrocarbon Solvents: Mediated by Antiglomerular Basement Membrane Antibody,” Arch En- 
viron Health, Vol. 25, 1972, pp. 365-369.

[4] E. T. O’Brien, W. O. Yeomn and J. A. E. Hobby, "Hepatorenal Damage from Toluene in 'Glue Sniffer', British Medical Journal, Vol. 2, 1971, pp. 29-30.

[5] S. M. Taher, R. J. Anderson, R. McCartney, M. M. Poputzer and R. W. Schirer, "Renal Tubular Acidosis Associated with Toluene 'Sniffing'," The New England Journal of Medicine, Vol. 290, No. 14, 1974, pp. 765-768. doi:10.1056/NEJM197404042901403

[6] A. H. Moss, P. A. Gabow and W. D. Kahney, “Fanconi’s Syndrome and Distal Renal Tubular Acidosis after Glue Sniffing,” Annals of Internal Medicine, Vol. 92, 1980, pp. 69-70.

[7] H. Z. Screicher, P. A. Gabow, A. H. Moss, D. Kono and W. D. Kaehny, "Syndrome of Toluene Sniffing in Adults," Annals of Internal Medicine, Vol. 94, 1981, pp. 758-762.

[8] G. Venkatamran, "Renal Damage and Glue Sniffing," British Medical Journal, Vol. 283, No. 6304, 1981, p. 1467. doi:10.1136/bmj.283.6304.1467-a

[9] W. E. Daniell, W. G. Couser and L. Rosenstock, “Occupational Solvent Exposure and Glomerulonephritis: A Case Report and Review of the Literature," Journal of the American Medical Association, Vol. 259, 1988, pp. 2280-2283. doi:10.1001/jama.259.15.2280

[10] C. M. Fored, G. Nise, E. Ejerblad, J. P. Fryzek, P. Lindblad, J. K. McLaughlin, C. G. Elinder and O. Nyren, "Absence of Association between Organic Solvent Exposure and Risk of Chronic Renal Failure: A Nationwide Population-Based Case-Control Study," Journal of the American Society of Nephrology, Vol. 15, No. 1, 2004, pp. 180-186. doi:10.1097/01.ASN.0000103872.60993.06

[11] U. Ravnskov, "Hydrocarbons May Worsen Renal Function Inglomerulonephritis: A Meta-Analysis of the CaseControl Studies," American Journal of Industrial Medicine, Vol. 37, No. 6, 2000, pp. 599-606.

doi:10.1002/(SICI)1097-0274(200006)37:6<599::AID-AJ IM4>3.3.CO;2-O

[12] S. S. Al-Ghamdi, D. A. Allen, M. J. Raftery and M. M. Yaqoob, "Organic Solvent-Induced Proximal Tubular Cell Toxicity via Caspase-3 Activation,” Clinical Toxicology, Vol. 41, No. 7, 2003, pp. 941-945. doi:10.1081/CLT-120026515

[13] S. S. Al-Ghamdi, M. J. Raftery and M. M. Yaqoob, "Acute Solvent Exposure Induced Activation of Cytochrome P4502E1 Causes Proximal Tubular Cell Necrosis by Oxidative Stress,” Toxicology in Vitro, Vol. 17, 2003, pp. 335-3412. doi:10.1016/S0887-2333(03)00021-3

[14] A.Sato, I. Yonekura, T. Kaneko and T. Koizumi, "Renal
Disorders Induced by Organic Solvents,” Sangyo Igaku, Vol. 30, No. 2, 1988, pp. 85-96.

[15] H. Savolainen, H. Vainio, M. Helojoki and E. Elovaara, "Biochemical and Toxicological Effects of Short-Term, Intermittent Xylene Inhalation Exposure and Combined Ethanol Intake,” Archives of Toxicology, Vol. 41, No. 3, 1978, pp. 195-205. doi:10.1007/BF00354091

[16] R. Lauwerys, A. Bernard, C. Viau and J. P. Buchet, "Kidney Disorders and Hematotoxicity from Organic Solvent Exposure," Scandinavian Journal of Work, Environment \& Health, Vol. 11, No. 2, 1985, pp. 83-90.

[17] J. M. Harrington, H. Whitby, C. N. Gray, F. J. Reid, T. C. Aw and J. A. Waterhouse, "Renal Disease and Occupational Exposure to Organic Solvents: A Case Referent Approach,” British Journal of Industrial Medicine, Vol. 46, No. 9, 1989, pp. 643-50.

[18] S. Jacob, M. He'ry, J. C. Protois, J. Rossert and B. Stengel, "Effect of Organic Solvent Exposure on Chronic Kidney Disease Progression: The GN-progress Cohort Study," Journal of the American Society of Nephrology, Vol. 18, No. 1, 2007, pp. 274-281. doi:10.1681/ASN.2006060652

[19] A. Mutti, T. Coccini, R. Alinovi, G. Toubeau, F. Broeckaert, E. Bergamaschi, P. Mozzoni, D. Nonclercq, A. Bernard and L. Manzo, "Exposure to Hydrocarbons and Renal Disease: An Experimental Animal Model," Ren Fail, Vol. 21, 1999, pp. 369-385. doi:10.3109/08860229909085101

[20] Van Der Laan, "Chronic Glomerulonephritis and Organic Solvents. A Case-Control Study," International Archives of Occupational and Environmental Health, Vol. 47, No. 1, 1980, pp. 1-8.

[21] P. Hotz, "Occupational Hydrocarbon Exposure and Chronic Nephropathy,” Toxicology, Vol. 90, No. 3, 1994, pp. 163-283. doi:10.1016/0300-483X(94)90091-4

[22] D. M. Hashimoto, K. T. Kelsey, T. Seitz, H. A. Feldman, B. Yakes and D. C. Christiani, "The Presence of Urinary Cellular Sediment and Albuminuria in Newspaper Pressworkers Exposed to Solvents," Journal of Occupational and Environmental Medicine, Vol. 33, No. 4, 1991, pp. 516-26.

[23] G. Remuzzi and T. Bertani, "Pathophysiology of Progressive Nephropathies," The New England Journal of Medicine, Vol. 339, 1998, pp. 1448-1456. doi:10.1056/NEJM199811123392007

[24] D. Solet and T. G. Robins, "Renal Function in Dry Cleaning Workers Exposed to Perchloroethylene," American Journal of Industrial Medicine, Vol. 20, No. 5, 1991, pp. 601-14. doi:10.1002/ajim.4700200504 